\title{
Savoirs locaux sur les marqueurs temporels en zone guinéenne au Togo
}

\author{
Kodjovi Mawuégnigan Léonard Agbodan ${ }^{(1)}$, Sêmihinva Akpavi ${ }^{(1)}$, Kossi Béssan \\ Amegnaglo ${ }^{(1)}$, Amah Akodewou ${ }^{(1,2)}$, Badabaté Diwediga ${ }^{(1)}$, Donko Koudzo Koda ${ }^{(1,3)}$, \\ Komlan Batawila $^{(1)}$, Koffi Akpagana ${ }^{(1)}$ \\ (1) Université de Lomé. Laboratoire de Botanique et Écologie Végétale (LBEV). 01 BP 1515. Lomé 01 (Togo). E-mail : \\ agbodana@gmail.com \\ (2) Université Paris-Saclay. AgroParisTech. F-75732 Paris (France). \\ (3) Université Felix Houphouët-Boigny. WASCAL. Changements climatiques et Biodiversité. BP 165. Abidjan 31 (Côte \\ d'Ivoire).
}

Reçu le 25 novembre 2019, accepté le 28 septembre 2020, mis en ligne le 8 octobre 2020.

Cet article est distribué suivant les termes et les conditions de la licence CC-BY (http://creativecommons.org/licenses/by/4.0/ deed.fr)

Description du sujet. La baisse et l'irrégularité des pluies modifient considérablement les saisons culturales, ce qui oblige les paysans à une restructuration du calendrier agricole en s'appuyant sur les savoirs locaux.

Objectifs. L'objectif de cette étude est de valoriser les savoirs locaux utilisés par les paysans dans la gestion du calendrier agricole. Les objectifs spécifiques sont, d'une part, d'inventorier les indicateurs biophysiques utilisés dans la définition des calendriers culturaux et, d'autre part, de déterminer les espèces végétales repères communes aux trois zones écologiques étudiées.

Méthode. Ce but fut atteint grâce à des interviews semi-directes individuelles et via des focus groups auprès d'un échantillon de 689 agriculteurs répartis dans 65 localités.

Résultats. Il ressort que la totalité des paysans enquêtés utilisent des marqueurs temporels dans la définition des calendriers culturaux, surtout dans la reconnaissance des débuts de saison pluvieuse (période de semis) et de saison sèche (période de récolte). Il s'agit principalement des indicateurs biologiques tels que la phénologie des plantes (en saison sèche : Triumfetta rhomboidea Jacq. [floraison], Spathodea campanulata P.Beauv. [floraison], Vitellaria paradoxa C.F.Gaertn. [fructification] et en saison pluvieuse : Millettia thonningii (Schum. \& Thonn.) Baker [floraison], V. paradoxa [floraison], Delonix regia (Hook.) Raf. [floraison], Ceiba pentandra (L.) Gaertn. [éclatement du fruit]). Au total, 67 espèces végétales ont été recensées comme indicateurs des variations des saisons agricoles. Outre les espèces végétales, la migration saisonnière des oiseaux (73\%), l'apparition des insectes (48\%), mais aussi des indicateurs abiotiques comme le sens du vent (100\%), les observations des étoiles $(82 \%)$ et le déplacement des nuages $(69 \%)$ sont utilisés comme repères dans la gestion des périodes agricoles.

Conclusions. La connaissance de ces signaux permet aux paysans d'anticiper sur d'éventuelles variabilités climatiques et suivre, diriger et adapter leurs activités culturales. Ces savoirs locaux constituent dès lors une aubaine pour l'adaptation aux changements climatiques.

Mots-clés. Marqueurs temporels, savoirs locaux, changements climatiques, adaptation, Togo.

\section{Local knowledge on temporal indicators in the Guinean zone of Togo}

Description of the subject. Important changes in cropping seasons caused by a decrease and erratic patterns in rainfall have forced farmers to redefine their agricultural calendars, which are based on local knowledge.

Objectives. The objective of this study was to promote local knowledge used in farming schedules. The specific objectives were to identify biophysical indicators used for planning cropping calendars, and to determine the target plant species common to all ecological zones.

Method. In total, 689 farmers distributed through 65 localities were surveyed through semi-direct interviews and focus group discussions.

Results. All respondent farmers reported relying on temporal markers for defining their cropping calendars, especially the recognition of the onset of the rainy season (sowing period) and rainfall cessation or the dry season (harvesting period). These 
campanulata P.Beauv.) and fruiting (Vitellaria paradoxa C.F.Gaertn.), which are used to indicate the beginning of the dry season. The flowering of Millettia thonningii (Schum. \& Thonn.) Baker, V.paradoxa, Delonix regia (Hook.) Raf. and the opening of ripened fruits (Ceiba pentandra [L.] Gaertn.) are used to identify the onset of the rainy season. A total of 67 species were identified as indicators of agricultural seasons. In addition to these plant species, the seasonal migration of birds (73\%), and of insects (48\%); and some abiotic indicators such as wind direction (100\%), star observations (82\%) and cloud movement $(69 \%)$ were reportedly used as landmarks in farming time management.

Conclusions. Knowledge about these signals is useful for anticipating possible climate variability based on traditional meteorological forecasting and for guiding local cropping calendars. This local knowledge regarding temporal markers is therefore relevant for agricultural adaptation to climate change.

Keywords. Temporal indicator, local knowledge, climate change, adaptation, Togo.

\section{INTRODUCTION}

Le changement climatique est un phénomène d'envergure mondiale dont les répercussions négatives inquiètent l'homme (Fatorić \& Morén-Alegret, 2013). Le domaine de l'agriculture, moteur clé du développement économique et d'éradication de la pauvreté dans les pays en voie de développement, est concerné car les activités agricoles demeurent intimement liées aux variabilités climatiques (FAO, 2016; Andersson et al., 2020). La baisse et l'irrégularité des pluies entrainent une variation du climat qui contraint les paysans à une restructuration de leurs calendriers culturaux. Ces derniers, moins informés sur les prévisions météorologiques nationales (Chisadza et al., 2013; Chisadza et al., 2014; Matewos, 2020), mobilisent leurs connaissances et compétences ainsi que les ressources disponibles pour s'adapter aux variabilités climatiques (Retnowati et al., 2014 ; Clifford et al., 2020). Le GIEC (2014) définit les savoirs locaux comme "les systèmes et pratiques du savoir autochtone, local et traditionnel, y compris la vision holistique qu'ont les peuples autochtones de leurs collectivités et de leur environnement». Ils constituent ainsi des repères de première importance utilisés pour s'adapter aux changements climatiques (Son et al., 2019). Un des atouts de ces savoirs réside dans la capacité à prédire des mutations saisonnières et constituerait ainsi un outil d'aide à la décision dans le processus d'adaptation au niveau local (Dounias \& Michon, 2013 ; Klein et al., 2014 ; Lewandowski et al., 2015).

Les savoirs locaux dont disposent les populations autochtones et qui contribuent aux diverses stratégies d'adaptation face au changement climatique leur ont permis depuis des temps immémoriaux de gérer durablement leur environnement d'une part et, d'autre part, de s'adapter aux aléas du changement climatique (Reyes-García et al., 2019). Ces savoirs et pratiques constituent le résultat d'observations millénaires, qui se transmettent oralement de génération en génération et qui s'étoffent au fil du temps afin d'agir face aux variations climatiques (Michon et al., 2017 ; Rasmus et al., 2020). Mais ces savoirs, qui naguère constituaient une pierre angulaire pour l'adaptation, sont marginalisés pour diverses raisons et pourraient manquer aux générations futures pour s'adapter aux changements climatiques (Alexander et al., 2011 ; Ngana et al., 2013). De nombreuses recherches scientifiques contribuent à identifier, documenter et analyser les savoirs ethnoclimatiques pour les intégrer aux données issues de la météorologie nationale (Cornell et al., 2019 ; Son et al., 2019 ; Andersson et al., 2020 ; Falconer et al., 2020 ; Matewos, 2020 ; Rasmus et al., 2020).

Le stade phénologique (fructification, feuillaison, défeuillaison, floraison et défloraison) de certaines espèces végétales, l'observation de l'abondance de certains types d'animaux (oiseaux, insectes, amphibiens) à une période de l'année, ainsi que le changement de direction de vent, constituent des informations capitales dont se servent les populations autochtones pour suivre, diriger et adapter leurs activités agricoles (Mhita, 2006; Dounias \& Michon, 2013; Udmale et al., 2014). La perception des indicateurs s'inscrit dans une logique de gestion adaptative qui permet aux paysans de gérer les saisons agricoles (Gyampoh et al., 2009 ; Sánchez-Cortés \& Chavero, 2011).

En Afrique, et au Togo en particulier, les recherches sur cette thématique restent très peu investiguées et sont encore fragmentaires malgré leur importance pour les populations autochtones et l'urgence climatique (Akpavi, 2008 ; Akpavi et al., 2013). Cette étude se propose de contribuer à la caractérisation, l'analyse et la valorisation des savoirs locaux sur les marqueurs biotemporels utilisés dans la zone guinéenne du Togo. Il s'agira spécifiquement :

- d'inventorier les indicateurs biophysiques utilisés dans la définition des calendriers culturaux ;

- de déterminer les espèces végétales communes dans les différentes zones écologiques prospectées.

\section{MATÉRIEL ET MÉTHODES}

\subsection{Description de la zone d'étude}

Le travail a été effectué dans la zone climatique guinéenne du Togo (Afrique de l'Ouest) qui s'étend de 
l'océan Atlantique au sud, jusqu'aux environs de la $8^{\text {è }}$ latitude nord (Figure 1). La zone guinéenne est couverte par trois zones écologiques notées III, IV et V (Ern, 1979) et compte près de 23 groupes ethnoculturels dont certains ont une aire de répartition géographique large. Sur le plan humain, trois grands groupes sont présents. Il s'agit des populations Ajatado au sud, Moyen-Mono à l'est et des plateaux à l'ouest représentant respectivement $44 \%, 3 \%$ et $4 \%$ de la population togolaise (Gayibor et al., 1997). Ces peuples ont développé depuis longtemps des systèmes de connaissances complexes, en lien étroit avec la nature, qui leur permettent de déceler les variations du climat au niveau local. Sur le plan de la végétation, la zone écologique III jouit d'un climat de type guinéen de plaine, caractérisé par une saison pluvieuse (de mars à octobre) et une saison sèche de quatre mois (de novembre à février). La pluviométrie et la température moyennes sont respectivement de $1200 \mathrm{~mm} \cdot \mathrm{an}^{-1}$ et $27^{\circ} \mathrm{C}$. La zone III représente le sanctuaire d'une agriculture basée essentiellement sur des cultures telles que le maïs, l'igname et le cotonnier (Akpavi, 2008). La zone écologique IV jouit d'un climat guinéen de montagne (Trochain, 1980) caractérisé par une grande saison pluvieuse (de mars à novembre) et une petite saison sèche de trois mois (de décembre à février), interrompue par une légère diminution de la pluviosité en aout. Les précipitations annuelles sont comprises entre 1400 et $1600 \mathrm{~mm} \cdot \mathrm{an}^{-1}$, tandis que la température annuelle varie de $25^{\circ} \mathrm{C}$ à $29^{\circ} \mathrm{C}$. Cette zone est surtout couverte par une mosaïque de formation végétale, des agroforêts, des forêts semi-décidues (Akpagana, 1989) avec comme cultures de rente le caféier, le cacaoyer et des cultures vivrières comme le manioc et le taro. La zone écologique $\mathrm{V}$ a un climat de type subéquatorial guinéen caractérisé par quatre saisons: une grande saison sèche (de novembre à février), une petite saison sèche (aout), une grande saison pluvieuse (de mars à juillet) et une petite saison pluvieuse (de septembre à octobre). Les précipitations sont comprises entre 800 et $950 \mathrm{~mm} \cdot \mathrm{an}^{-1}$, tandis que les températures annuelles varient entre $27^{\circ} \mathrm{C}$ et $30^{\circ} \mathrm{C}$. Cette zone est connue pour sa grande diversité en produits agricoles tels que le cocotier, le palmier à huile, le maïs, le manioc et le piment.

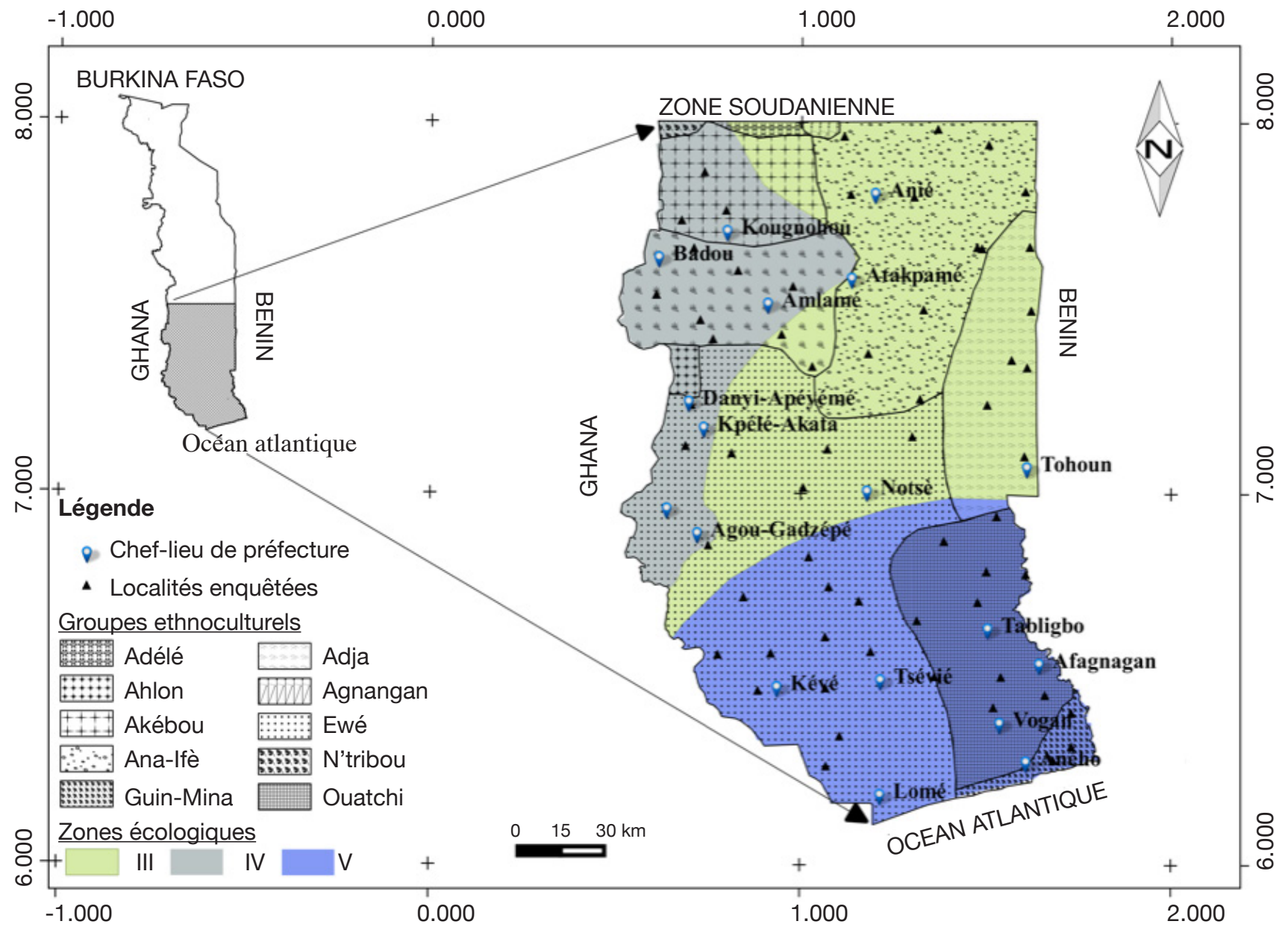

Figure 1. Localités enquêtées et localisation de la zone d'étude - Investigated localities and location of the study area. 


\subsection{Collecte des données}

Les localités investiguées ont été déterminées sur base d'un choix raisonné et non aléatoire de villages au sein de 11 unités géographiques majoritaires de la zone d'étude (Akpavi, 2008 ; Kebenzikato et al., 2015). Par définition, une unité correspond à l'aire géographique d'une ethnie dans une zone écologique (Figure 1). Une enquête exploratoire a été réalisée, d'une part pour évaluer le questionnaire et, d'autre part, pour déterminer la taille de l'échantillon à investiguer et pour l'amélioration de certaines rubriques dudit questionnaire (Tableau 1). La taille de l'échantillon des enquêtés a été déterminée en intégrant les données de l'enquête exploratoire dans la formule de Dagnelie (1998) :

$$
N=\frac{U_{1-\alpha / 2}^{2} \times p(1-p)}{d^{2}}
$$

Dans cette formule, $N$ est la taille minimale de l'échantillon à déterminer, $p$ est la proportion d'enquêtés ayant des connaissances sur les indicateurs biophysiques de dégradation du sol, $U_{1-\alpha / 2}$ est la valeur $(1,96)$ de la loi normale à la valeur de probabilité 1- $\alpha / 2$ avec $\alpha=5 \%, d$ est la marge d'erreur de l'estimation fixée à une valeur de $5 \%$ (Agbodan et al., 2019; Agbodan et al., 2020).

Au total, 689 enquêtés issus de 65 localités ont été prospectés entre septembre 2017 et mars 2018. Les ethnies Ewé, Adja et Kabyè dominent dans l'échantillon de par leurs larges répartitions géographiques dans la zone d'étude (Gayibor et al., 1997). La majorité des enquêtés avait pour activité principale l'agriculture et avait un âge compris entre [30-60 ans]. Les données ont été collectées via des enquêtes semi-structurées individuelles et par focus group (Twumasi, 2001 ; Akpavi et al., 2013). Les points géographiques des localités prospectées ont été enregistrés à l'aide d'un GPS. Les principales rubriques du questionnaire sont relatives aux connaissances environnementales locales utilisées pour la planification et l'exécution des activités agricoles. Les indicateurs locaux déterminant

Tableau 1. Statistiques sur les localités investiguées Statistics on investigated localities.

\begin{tabular}{llll}
\hline & Zone III & Zone IV & Zone V \\
\hline$p$ & 0,81 & 0,9 & 0,75 \\
Taille minimale (N) & 237 & 139 & 289 \\
Taille réelle & 244 & 145 & 300 \\
\hline Nombre de localités & 30 & 12 & 23 \\
Groupes ethnoculturels & 9 & 6 & 5 \\
\hline
\end{tabular}

les cycles saisonniers de début de saisons pluvieuse et sèche ont été recensés auprès de chaque personne interviewée.

\subsection{Traitement et analyse des données}

Les données collectées ont été saisies et organisées dans un tableur Microsoft Excel 2016, de manière à avoir deux matrices: "espèces végétales repères $x$ zones écologiques » et «espèces végétales repères $x$ stade phénologique ». La nomenclature botanique utilisée pour l'identification des espèces végétales (ligneuses et herbacées) est celle de Brunel et al. (1984). La première matrice a servi pour une AFC (Analyse Factorielle de Correspondance) et a permis de déterminer les espèces végétales communes aux trois zones écologiques et celles citées comme repère dans une seule zone écologique. La deuxième matrice a permis une analyse descriptive par histogramme. Le logiciel R, grâce à son interface Rcmdr, a permis la réalisation des différentes analyses et des graphiques.

\section{RÉSULTATS}

\subsection{Diversité des marqueurs utilisés par les paysans dans la gestion du temps}

Dans la zone d'étude, la totalité des paysans enquêtés utilisent les marqueurs temporels dans la structuration des agendas agricoles, notamment la reconnaissance des débuts de saison pluvieuse (période de semis) et de saison sèche (période de récolte). Il s'agit principalement d'indicateurs biologiques (espèces végétales, migration des oiseaux, chant des amphibiens) et abiotiques (sens du vent, observations des étoiles, déplacement des nuages) (Figure 2). La fructification $(100 \%)$ et la floraison $(100 \%)$ des espèces végétales sont également des paramètres biologiques unanimement utilisés par les ménages investigués. Parmi les facteurs abiotiques, les vents dominants (le sens et l'intensité) et l'observation des étoiles (présence, absence, abondance, brillance, formation d'une masse d'étoiles) sont les plus utilisés.

\subsection{Indicateurs végétaux}

Les paysans se basent essentiellement sur des phases phénologiques (fructification, floraison, feuillaison, défloraison, défeuillaison) des plantes pour se repérer dans le temps. En somme, 67 espèces végétales (voir Annexe) réparties en 64 genres et 28 familles ont été recensées lors des enquêtes. La figure 3 illustre la fréquence relative des 25 espèces végétales « repères » ayant une forte fréquence de citation. L'analyse du graphe montre une prédominance d'un faible nombre 


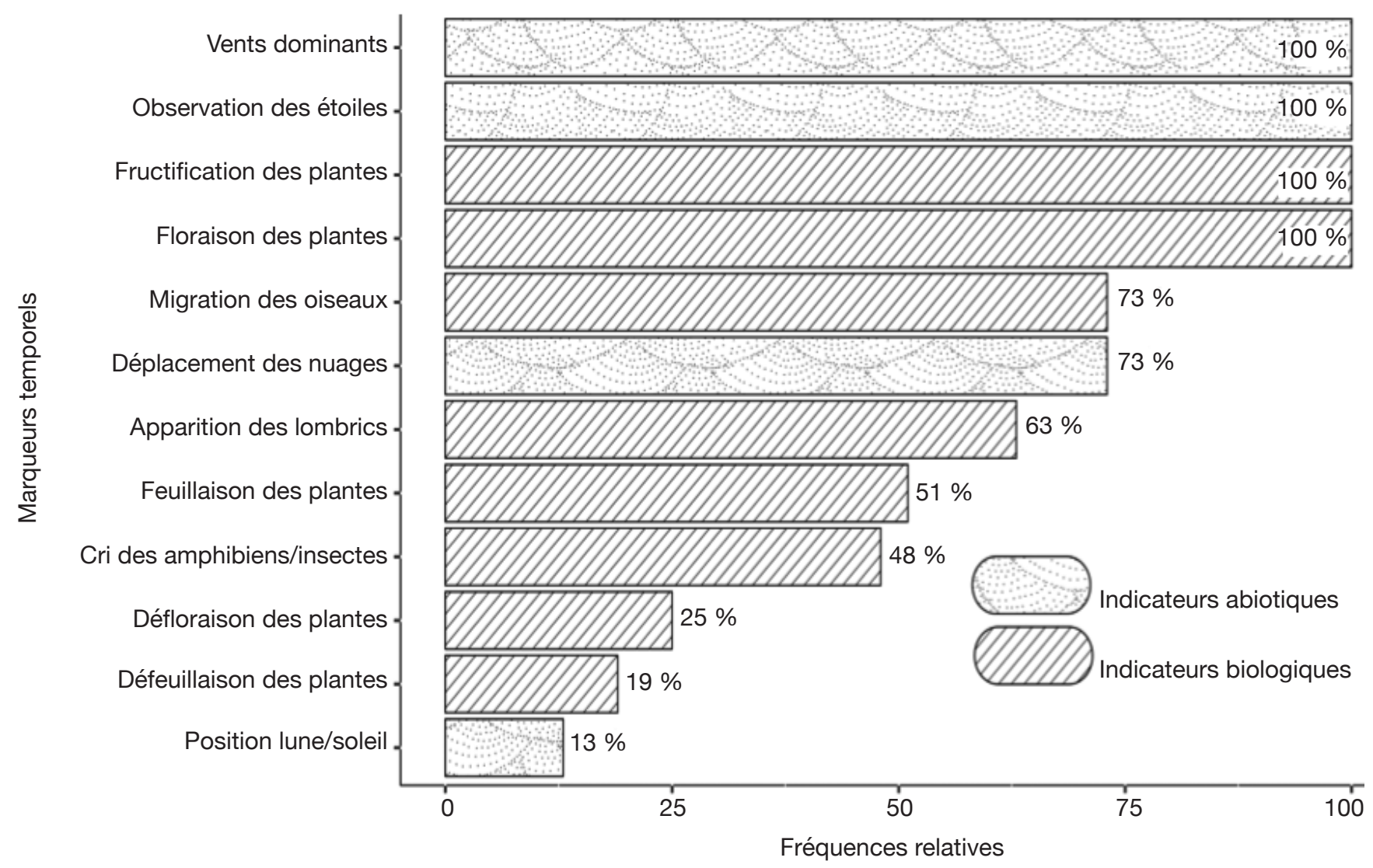

Figure 2. Indicateurs utilisés par les paysans dans la définition des calendriers agricoles - Indicators used by farmers in defining agricultural calendars.

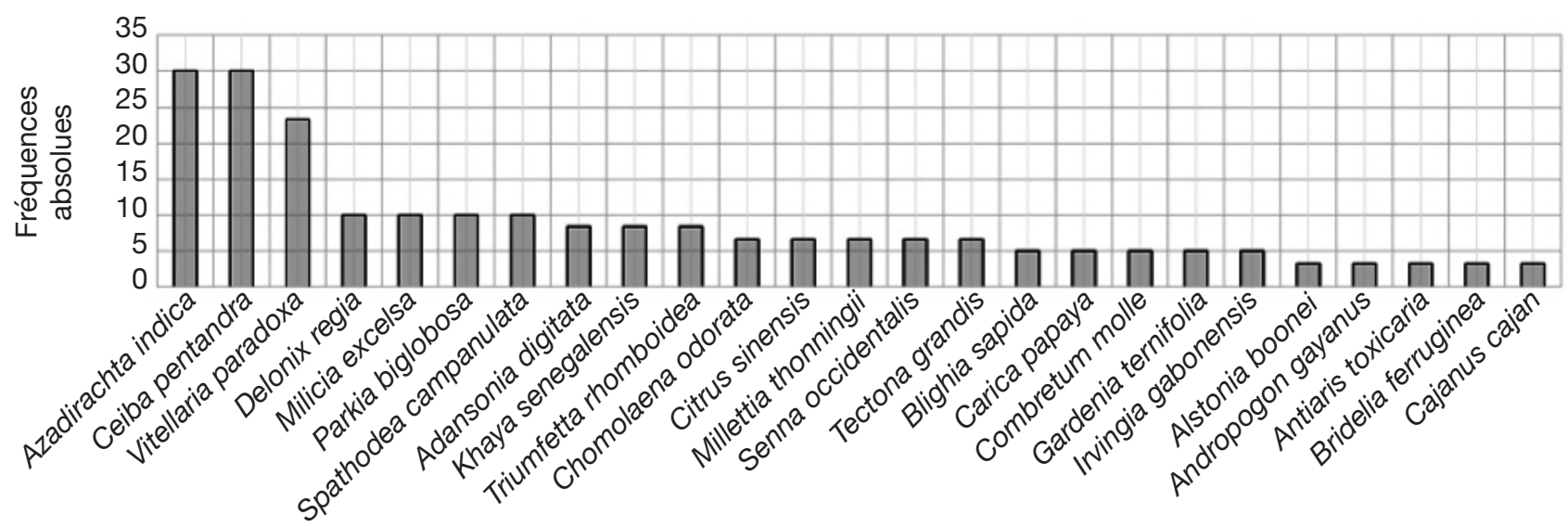

Figure 3. Fréquences des 25 espèces végétales les plus citées comme marqueurs temporels - Frequencies of the 25 most cited vegetal species as time markers. 
d'espèces à forte fréquence et celle d'un grand nombre d'espèces de faible à moyenne fréquence.

\subsection{Espèces végétales indicatrices de saison culturale suivant les zones écologiques}

L'AFC ci-dessous regroupe les espèces végétales citées dans les zones écologiques et celles communes aux trois zones écologiques (Figure 4). L'analyse $\mathrm{du}$ graphique montre que les espèces végétales Azadirachta indica A.Juss. (Meliaceae), Delonix regia (Hook.) Raf. (Ceasalpiniaceae), Millettia thonningii (Schum. \& Thonn.) Baker (Papillonaceae), Vitellaria paradoxa C.F.Gaertn (Sapotaceae), Milicia excelsa (Welw.) C.C.Berg (Moraceae), Ceiba pentandra (L.) Gaertn. (Bombacaceae), Khaya senegalensis (Desv.) A.Juss. (Meliaceae) et Spathodea campanulata P.Beauv. (Bignoniaceae) constituent les marqueurs temporels végétaux utilisés comme indicateurs dans les trois zones écologiques prospectées.

\subsection{Espèces végétales indicatrices du début de la saison pluvieuse}

$\mathrm{Au}$ total, 44 espèces végétales sur les 67 recensées servent d'indicateurs de saison pluvieuse (Annexe). La figure 5 résume les paramètres phénologiques des espèces indicatrices de saison pluvieuse. Par exemple, la floraison des espèces comme $M$. thonningii et $G$. kola constitue un signal avant-coureur (forte fréquence de citation) des premières pluies, elles-mêmes à l'origine du déclenchement des cycles agricoles dans la zone guinéenne du Togo. La floraison de $D$. regia, Mangifera indica L., Adansonia digitata L., Aspilia rudis Oliv. \& Hiern; la fructification d'A. indica, Irvingia gabonensis (Aubry-Lecomte ex O'Rorke) Baill. et de Combretum molle R.Br. ex G.Don, puis la feuillaison de Dioscorea cayennensis Lam. et de Jatropha curcas L. indiquent aussi l'arrivée de la saison pluvieuse. L'éclatement des fruits de $C$. pentandra, l'assèchement des fruits de Triumfetta rhomboidea Jacq. signalent la saison des pluies.

Il ressort qu'une bonne saison peut être aussi prédite grâce aux indicateurs suivants: une longue période intense de sècheresse (28\%), l'arrivée de la première pluie dans le mois de janvier (57\%) et surtout, une forte production fruitière $(81 \%)$ du manguier (Mangifera indica L.) et du néré (Parkia biglobosa [Jacq.] G.Don). Ces connaissances basées sur les éléments de la nature favorisent l'adaptation des calendriers culturaux aux changements climatiques.

\subsection{Espèces végétales indicatrices du début de la saison sèche}

Au total, 38 espèces végétales sont utilisées comme indicateurs de saison sèche (Figure 6). La floraison des espèces végétales telles que T.rhomboidea et

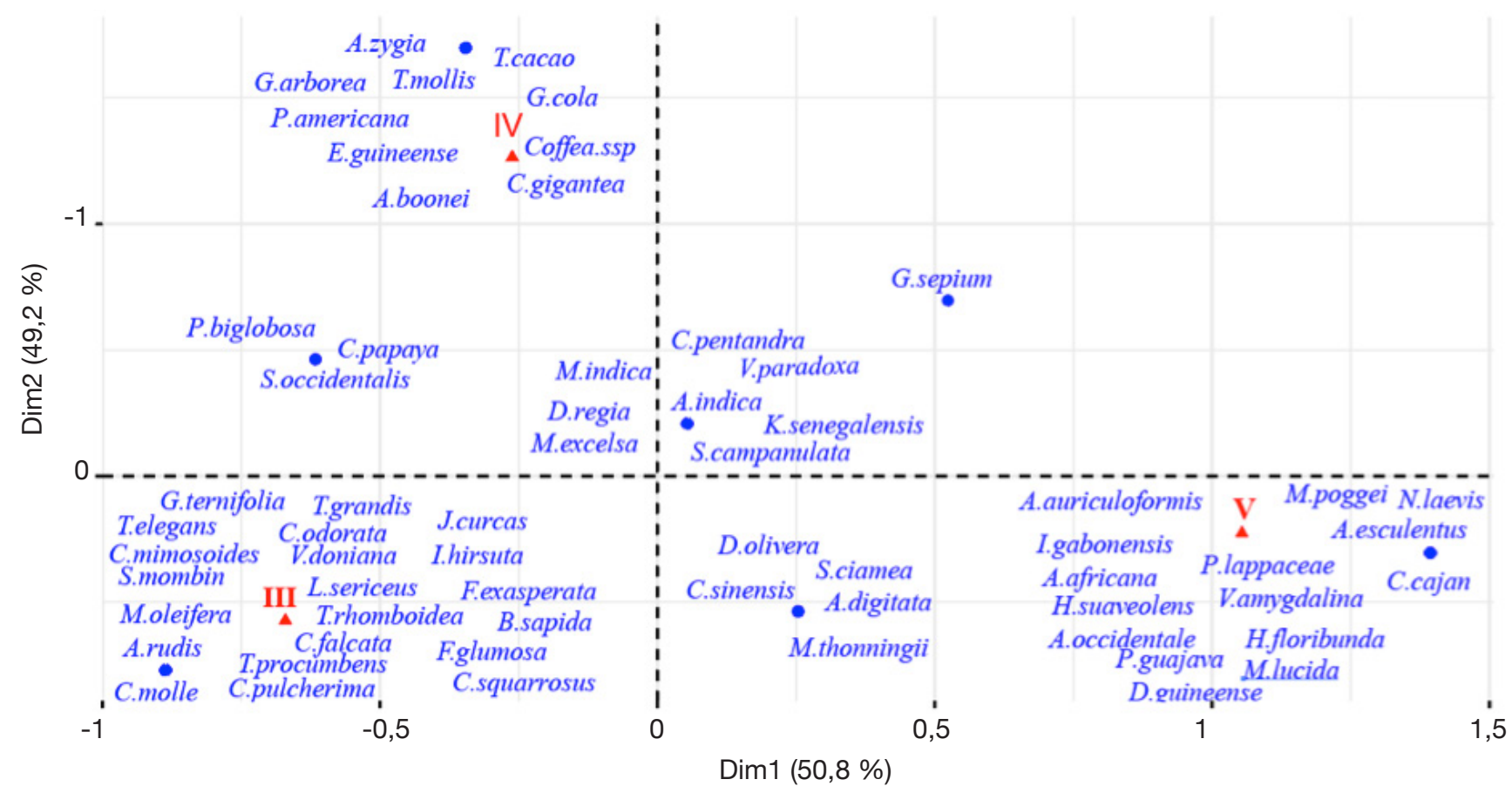

Figure 4. Analyse factorielle de correspondance entre les espèces végétales utilisées comme marqueurs temporels et les zones écologiques (III, IV et V) - Correspondence factor analysis between plant species used as temporal markers and ecological zones (III, IV and V). 


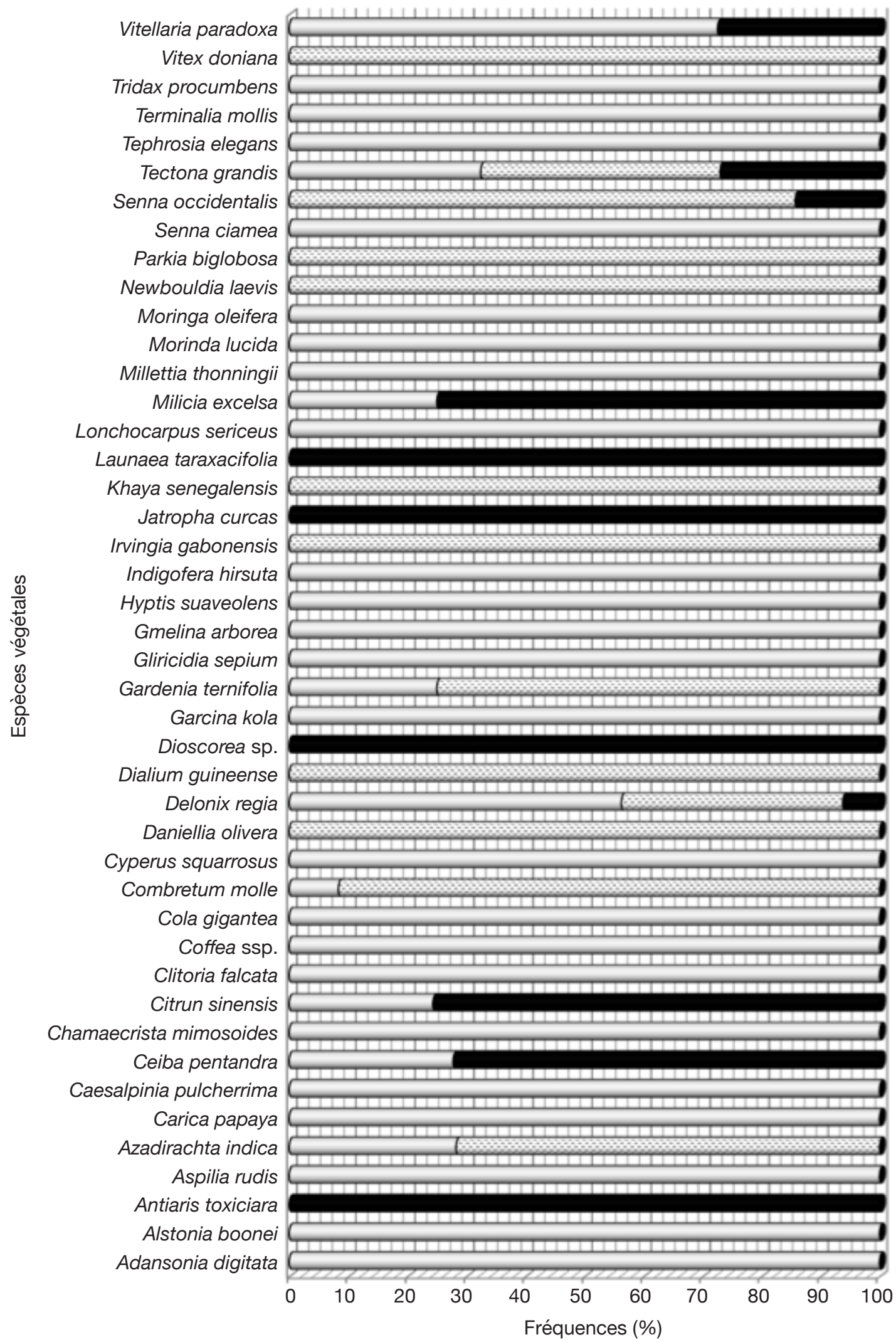

Floraison a Fructification $\mathbf{E}$ Feuillaison

Figure 5. Phénologie des espèces indicatrices de début de saison pluvieuse - Phenology of indicator plants of early rainy season. 


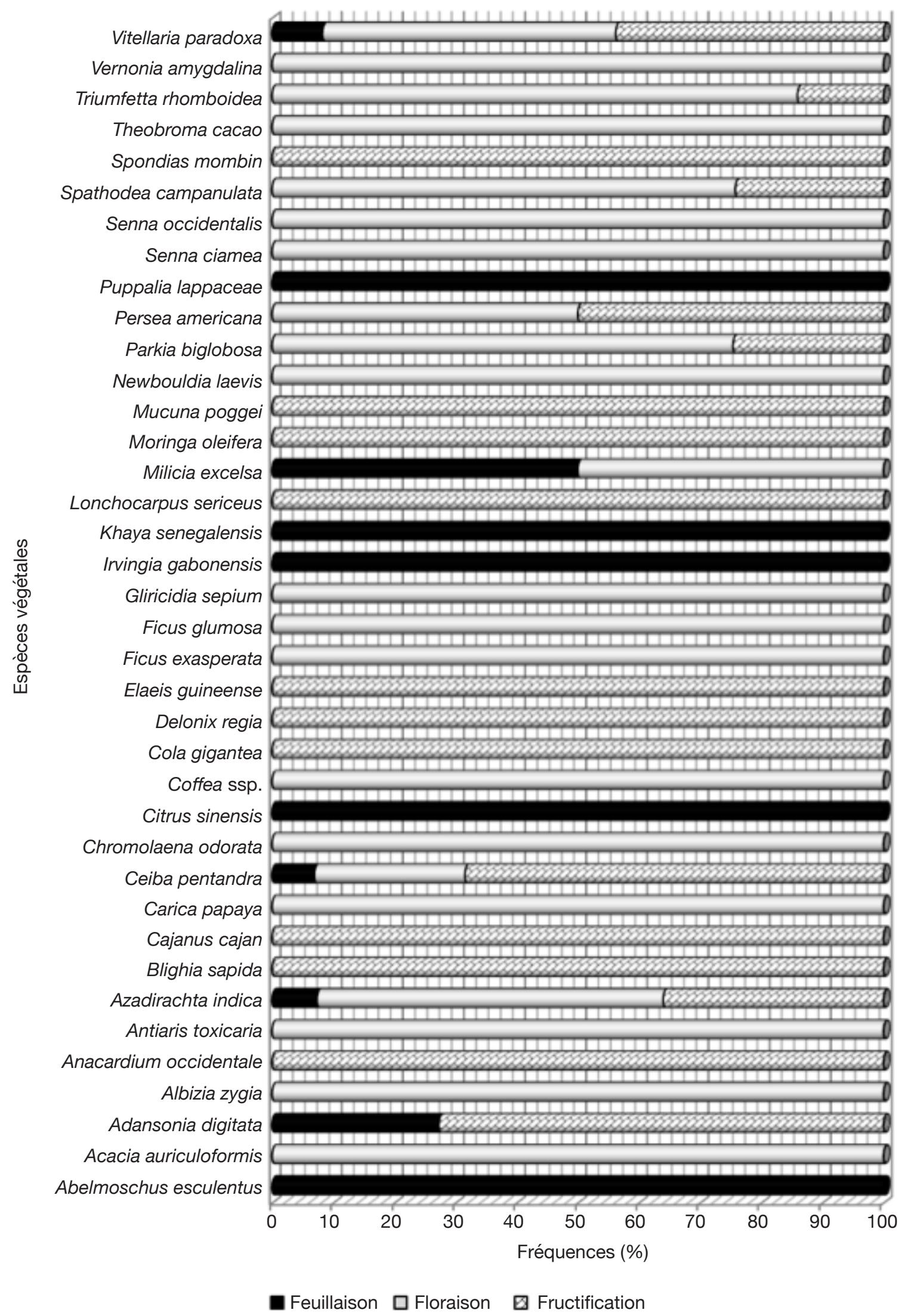

Figure 6. Phénologie des espèces indicatrices de début de saison sèche - Phenology of indicator plants of early dry season. 
S. campanulata constitue des signaux de début de saison sèche respectivement dans les zones écologiques III et IV de la zone guinéenne du Togo. Cette période correspond aux pratiques des travaux à caractère non agricole tels que la fabrication du vin de palme, la chasse, etc. La fructification d'A. digitata indique aux paysans la fin des récoltes et le début de la saison sèche. La sècheresse est aussi annoncée par la feuillaison de K. senegalensis et d'A.digitata. Certaines espèces végétales appartiennent à deux catégories phénologiques. À titre d'exemple, la floraison et la feuillaison synchrone de M. excelsa annoncent la saison sèche. Ces différents paramètres constituent des repères permettant aux paysans de définir et de s'adapter efficacement aux calendriers agricoles.

Les dates de début de saison sèche sont aussi reconnues par les populations grâce à la défloraison de M.thonningii, Gomphrena celosioides Mart. et de Citrus limon (L.) Osbeck.

\subsection{Indicateurs animaux}

L'apparition de certaines espèces animales à une période précise de l'année fournit des informations nécessaires aux paysans dans la définition du calendrier agricole. La saison des pluies commence, chez les autochtones, par l'observation en abondance des vers anéciques tels que le lombric (18\% des enquêtés) dans les champs et jachères; des oiseaux comme le héron pique bœuf (Bubulcus ibis, $37 \%$ des enquêtés); le coucal du Sénégal (Centropus senegalensis, $21 \%$ des enquêtés) et l'hirondelle (Hirundo rustica, $37 \%$ des enquêtés) autour des ménages; des chants des amphibiens tels que le crapaud (19\% des enquêtés) et des insectes comme la cigale (9\% des enquêtés) la nuit. La migration précitée (la non-observation ou la raréfaction) des espèces animales signale le début de la saison sèche. L'indicateur phare marquant la saison sèche reste l'observation de l'épervier (Milvus migrans, $84 \%$ ) autour des ménages.

\section{DISCUSSION}

Les enquêtes ethno-climatologiques réalisées sur les marqueurs temporels permettent de retenir que l'apparition de certains animaux et insectes, le chant des amphibiens et oiseaux, ainsi que les observations abiotiques de type climatique et astronomique basées sur les nuages, le soleil et la lune sont des indicateurs qui permettent aux paysans de se repérer dans le temps par anticipation et donc de mieux gérer leurs calendriers agricoles. Ces résultats sont similaires à ceux obtenus par Audefroy \& Sánchez (2017) au Mexique, où ces indicateurs permettent non seulement de guider la prise de décision des itinéraires culturaux, mais aussi de prédire le risque des évènements extrêmes tels que les catastrophes naturelles comme les inondations, les séismes et les longues périodes sèches. Ces connaissances constituent un savoir propre et permettent aux populations autochtones de comprendre les conditions climatique et météorologique au niveau local avant toute prise de décision en matière de cultures et de pratiques agricoles (Anandaraja et al., 2008; Kalanda-Joshua et al., 2011 ; Rautela \& Karki, 2015).

Parmi les indicateurs recensés, la fructification et la floraison des plantes restent les variables phénologiques les plus utilisées dans la définition des calendriers culturaux. Les études de Cleland et al. (2007) et de Rosenzweig et al. (2008) ont démontré que des changements phénologiques sont inextricablement liés aux variations climatiques et peuvent, dès lors, servir d'évènements clés pour les populations locales. En effet, la variation des périodes de floraison, de fructification et de feuillaison des plantes dépend de facteurs intrinsèques et extrinsèques à la plante (Nguemo et al., 2004 ; Mallard, 2016). Parmi les variables extrinsèques, le climat (humidité relative, température minimale, etc.) joue un rôle prépondérant dans l'apparition des évènements périodiques (phénologie) chez la plante. Ainsi, chez les peulhs au Burkina Faso, le comportement des arbres fruitiers en certaines périodes de l'année est considéré comme le «seul indicateur infaillible de saison», qui soit vérifiable d'année en année (Bergeret, 2002). La forte fructification (par rapport à la normale) de M. indica, indicatrice de bonne saison culturale selon le présent travail, confirme les travaux de Kalanda-Joshua et al. (2011) au Malawi. Divers exemples similaires sur la forte production fruitière des espèces végétales indicatrice d'une bonne saison culturale sont observés par Bergeret et al. (2002) au Burkina Faso, Chisadza et al. (2013) au Zimbabwe et Chang'a et al. (2010) en Tanzanie.

Les enquêtes ont permis de recenser 67 espèces végétales utilisées comme repères dans la gestion du temps dans la zone guinéenne du Togo. Cette diversité implique que les populations se basent principalement sur les plantes présentes dans leurs milieux immédiats pour se repérer. La diversité des plantes utilisées comme repères est de 5 en Tanzanie (Chang'a et al., 2010) et 11 au Zimbabwe (Chisadza et al., 2013). La forte diversité de plantes indicatrices obtenue par le présent travail serait liée aux fortes diversités physique, édaphique, écologique, climatique, pédologique, écosystémique et ethnoculturelle présentes dans la zone d'étude (Ern, 1979 ; Trochain, 1980 ; Faure \& Pennanaech, 1981 ; White, 1983 ; Brunel et al., 1984 ; Addra \& Fahem, 1994 ; Gayibor et al., 1997 ; Agbossoumonde, 1998 ; Gnazou, 2008). L'AFC réalisée sur les plantes utilisées comme marqueurs temporels a permis de déterminer les espèces végétales communes et celles citées dans 
une seule zone écologique. Les espèces végétales citées comme repères dans la zone écologique IV constituent les espèces agroforestières des plantations de café et de cacao (Koda et al., 2016), tandis que celles recensées dans les zones écologiques III et V correspondent aux espèces de jardins de cases et espèces fruitières laissées dans les champs (Kebenzikato et al., 2014).

Hormis les indicateurs biotiques végétaux, le chant et l'apparition des oiseaux, insectes et amphibiens sont des paramètres très importants aux yeux des paysans pour l'adaptation aux variations saisonnières et climatiques (Mhita, 2006). Les travaux de Lewandowski et al. (2015) démontrent que les insectes constituent des indicateurs biotemporels particulièrement remarquables, car ils sont en mesure de réagir à d'infimes altérations climatiques que les humains sont incapables de ressentir directement. Nos résultats sont similaires à ceux d'Andersson et al. (2020) en Afrique du Sud, qui démontraient que l'observation d'un grand nombre de vers de terre Sifenenefene (langue locale) et des chants d'oiseaux de Hlahlamedupe (langue locale) en octobre/novembre servait de critère fiable aux paysans pour anticiper l'approche imminente de la saison des pluies.

\section{CONCLUSIONS}

Cette étude a montré que des marqueurs temporels sont utilisés par les paysans pour définir les saisons agricoles. Ces indicateurs (biotique et abiotique) constituent des savoirs locaux qui ont une valeur culturelle clé, car ces savoirs permettent aux populations de prendre en compte le changement dans leurs pratiques quotidiennes, d'y répondre avec de nouvelles pratiques et donc de s'adapter à la variabilité climatique et aux changements en général. Ces connaissances sont mises en pratique, acceptées et s'adaptent au cours des générations; de ce fait, elles cristallisent le vécu et les représentations des populations autochtones sur leur environnement dans ce contexte de changement climatique. La synergie de l'observation des différents indicateurs permet aux paysans de mieux planifier les saisons agricoles et de présager d'éventuels désastres climatiques. Parmi les indicateurs inventoriés, la phénologie des espèces végétales reste l'indicateur le plus utilisé, car elle reste l'indicateur facilement observable par les paysans. Les résultats montrent que plusieurs espèces végétales repères ne sont pas communes aux trois zones écologiques, indiquant une diversification des savoirs au sein des différents groupes ethnoculturels. Au total, huit espèces végétales sont citées dans toutes les zones écologiques et servent à planifier les activités rurales. Il s'agit de A. indica, C.pentandra, D. regia, K. senegalensis, $M$. excelsa, $M$. indica, S. campanulata et $V$. paradoxa. Les savoirs locaux basés sur les éléments de la nature doivent être analysés, quantifiés et intégrés aux pools de données météorologiques scientifiques. Grâce à des investigations écologiques régulières, il sera possible d'établir une corrélation entre les savoirs locaux et les savoirs scientifiques. À cette étape, la description et la vulgarisation des savoirs locaux indispensables à l'adaptation aux changements climatiques est nécessaire afin qu'ils soient partagés et conservés dans les différents groupes ethnoculturels au bénéfice de la population.

\section{Remerciements}

Les auteurs expriment leur gratitude aux organismes IFS (Fond International pour la Science), le COMSTECH (Coopération Scientifique et Technologique) et IDEA WILD pour l'appui matériel et financier. Nous remercions vivement le Laboratoire de Botanique et Écologie Végétale (LBEV) pour avoir prêté son cadre de travail et aux populations locales qui ont accepté de livrer gratuitement leurs connaissances propres pour la science.

\section{Bibliographie}

Addra T. \& Fahem A., 1994. Atlas du développement régional du Togo. Lomé : Editogo.

Adjossou K., 2009. Diversité, structure et dynamique de la végétation dans les fragments de forêts humides $d u$ Togo : les enjeux pour la conservation de la biodiversité. Thèse de doctorat : Université de Lomé (Togo).

Agbodan K.M.L. et al., 2019. Connaissances écologiques locales sur les indicateurs de dégradation des sols utilisées par les paysans dans la zone guinéenne du Togo (Afrique de l'ouest). Sci. Vie Terre Agron., 1, 47-55.

Agbodan K.M.L. et al., 2020. Description agromorphologique et détermination du potentiel antioxydant des variétés sous-utilisées et nouvellement introduites de maïs, manioc, niébé et piment dans la région maritimeEst du Togo. Afr. J. Food Agric. Nutr. Dev., 20(3), 15936-15953.

Agbossoumonde Y., 1998. Les complexes ultrabasiquesbasiques de la chaine panafricaine au Sud-Togo: pétrologie et implications géodynamiques. Thèse de doctorat : Université de Saint-Étienne (France).

Akpagana K., 1989. Recherches sur les forêts denses humides du Togo. Thèse d'État : Bordeaux III (France).

Akpavi S.,2008. Plantes alimentaires mineures ou menacées de disparition au Togo: diversité, ethnobotanique et valeurs. Thèse de doctorat : Université de Lomé (Togo).

Akpavi S. et al., 2013. Valeur socio-culturelle des plantes alimentaires : un facteur de préservation. Eur. Sci.J., 9, 383-395.

Alexander C. et al., 2011. Linking indigenous and scientific knowledge of climate change. BioScience, 61(6), 477484, doi.org/10.1525/bio.2011.61.6.10 
Anandaraja N. et al., 2008. Indigenous weather and forecast practices of Coimbatore district farmers of Tamil Nadu. Indian J. Trad. Knowl., 7, 630-633.

Andersson L. et al., 2020. Local early warning systems for drought-Could they add value to nationally disseminated seasonal climate forecasts? Weather Clim. Extremes, 28, 100241, doi.org/10.1016/j.wace.2019.100241

Audefroy J.F. \& Sánchez B.N.C., 2017. Integrating local knowledge for climate change adaptation in Yucatán, Mexico. Int. J. Sustainable Built Environ., 6, 228-237, doi.org/10.1016/j.ijsbe.2017.03.007

Bergeret A., 2002. Saisons mouvantes, prévisions, présages et décision chez les peuls du Yatenga (Burkina Faso). Paris : IRD Éditions, 213-232.

Brunel J., Hiepko P. \& Scholz H., 1984. Flore analytique du Togo. Phanérogames. Eschorn, Allemagne : GTZ.

Chang'a L.B., Yanda P.Z. \& Ngana J., 2010. Indigenous knowledge in seasonal rainfall prediction in Tanzania: a case of the south-western Highland of Tanzania. J. Geogr. Regional Planning, 3, 66-72.

Chisadza B., Tumbare M., Nhapi I. \& Nyabeze R.W., 2013. Useful traditional knowledge indicators for drought forecasting in the Mzingwane catchment area of Zimbabwe. Disaster Prev. Manage., 22, 312-325, doi. org/10.1108/dpm-10-2012-0109

Chisadza B., Tumbare M., Nyabeze R. \& Nhapi W.I., 2014. Validation of local knowledge drought forecasting systems in the Limpopo River Basin in Southern Africa. Disaster Prev. Manage., 23, 551-566, doi.org/10.1108/ dpm-02-2014-0032

Cleland E.E. et al., 2007. Shifting plant phenology in response to global change. Trends Ecol. Evol., 22, 357-365.

Clifford K.R., Travis W.R. \& Nordgren L.T., 2020. A climate knowledges approach to climate services. Clim. Serv., 1-8, doi.org/10.1016/j.cliser.2020.100155

Cornell J.D., Quintas-Soriano C., Running K. \& Castro A.J., 2019. Examining concern about climate change and local environmental changes from an ecosystem service perspective in the Western US. Environ. Sci.Policy, 101, 221-231, doi.org/10.1016/j.envsci.2019.08.021

Dagnelie P., 1998. Statistique théorique et appliquée. Tome 2. Inférence statistique à une et à deux dimensions. Bruxelles : De Boeck Université.

Dounias E. \& Michon G., 2013. Sentimiel. Des abeilles et des hommes : savoirs locaux naturalistes, apicollecte et changement global. In : Actes du colloque «Petits et percutants : des projets de recherche sur la biodiversité », 4 décembre 2013, Maison de la Chimie, Paris, France. Paris : Fondation pour la Recherche sur la Biodiversité, 68.

Ern H., 1979. Die Vegetation Togos. Gliederung, Gefährdung, Erhaltung. Willdenowia, 9, 295-315.

Falconer L. et al., 2020. The importance of calibrating climate change projections to local conditions at aquaculture sites. Aquaculture, 514, 734487, doi. org/10.1016/j.aquaculture.2019.734487
FAO, 2016. Minimum dietary diversity for women: a guide for measurement. Roma: FAO.

Fatorić S. \& Morén-Alegret R., 2013. Integrating local knowledge and perception for assessing vulnerability to climate change in economically dynamic coastal areas: the case of natural protected area Aiguamolls de l'Empordà, Spain. Ocean Coastal Manage., 85, 90-102, doi.org/10.1016/j.ocecoaman.2013.09.010

Faure P. \& Pennanaech B., 1981. Atlas du Togo. Paris : Éditions Jeune Afrique.

Gayibor N.L. et al., 1997. Histoire des Togolais : 1. Des origines à 1884. Lomé : Presses de l'Université du Bénin.

GIEC, 2014. Changements climatiques 2014 ; rapport de synthèse : contribution des Groupes de travail I, II et III au cinquième Rapport d'évaluation du Groupe d'experts intergouvernemental sur l'évolution du climat. Genève, Suisse : GIEC.

Gnazou M.D.T, 2008. Étude hydrodynamique, hydrochimique, isotopique et modélisation de l'aquifère $d u$ bassin sédimentaire côtier du Togo. Thèse de doctorat : Université de Lomé (Togo).

Gyampoh B., Amisah S., Idinoba M. \& Nkem J., 2009. Using traditional knowledge to cope with climate change in rural Ghana. Unasylva, 60, 70-74.

Kalanda-Joshua M., Ngongondo C., Chipeta L. \& Mpembeka F., 2011. Integrating indigenous knowledge with conventional science: enhancing localised climate and weather forecasts in Nessa, Mulanje, Malawi. Phys. Chem. Earth, Parts A/B/C, 36, 996-1003, doi. org/10.1016/j.pce.2011.08.001

Kebenzikato A.B. et al., 2014. Distribution et structure des parcs à Adansonia digitata L. (baobab) au Togo (Afrique de l'Ouest). Afr. Sci., 10, 434-449.

Kebenzikato A.B. et al., 2015. Connaissances ethnobotaniques du baobab (Adansonia digitata L.) au Togo. Biotechnol. Agron. Soc. Environ., 19, 247-261.

Klein J.A. et al., 2014. Unexpected climate impacts on the Tibetan Plateau: local and scientific knowledge in findings of delayed summer. Global Environ. Change, 28, 141-152, doi.org/10.1016/j.gloenvcha.2014.03.007

Koda D.K., Adjossou K., Djego J.G. \& Guelly K.A., 2016. Diversité et usages des espèces fruitières des systèmes agroforestiers à caféiers du Plateau-Akposso au Togo. Afr. Sci., 12, 113-119.

Lewandowski S. et al., 2015. Des savoirs locaux revisités. In: Reinert M. et al. Changement climatique: quels défis pour le Sud? Marseille, France : IRD, 219-231.

Mallard F., 2016. Programme les sentinelles du climat. Tome I. Développement d'indicateurs des effets du changement climatique sur la biodiversité en NouvelleAquitaine. Le Haillan, France : Cistude Nature.

Matewos T., 2020. The state of local adaptive capacity to climate change in drought-prone districts of rural Sidama, southern Ethiopia. Clim. Risk Manage., 27, 1-12, doi.org/10.1016/j.crm.2019.100209 
Mhita M.S., 2006. Training manual traditional knowledge for nature an environmental conservation, agriculture, food security and disaster management in Tanzania. Dar es Salaam, Tanzania: Tanzania Meteorological Agency, 42.

Michon G., Berriane M., Romagny B.\& Alifriqui M., 2017. Les savoirs locaux peuvent-ils inspirer des solutions adaptatives dans les arrière-pays du Maroc. HespérisTamuda, 52, 319-356.

Ngana F., Ababa A.M., Gapia M. \& Kossi L., 2013. Météorologie traditionnelle et activités rurales chez les Mandja de Sibut, République Centrafricaine. Geo-EcoTrop, 37, 303-312.

Nguemo D.D. et al., 2004. Inventaire et identification des plantes mellifères de la zone soudano-guinéene d'altitude de l'Ouest Cameroun. Tropicultura, 22, 139-145.

Rasmus S. et al., 2020. Climate change and reindeer management in Finland: co-analysis of practitioner knowledge and meteorological data for better adaptation. Sci. Total Environ., 710, 136229, doi.org/10.1016/j. scitotenv.2019.136229

Rautela P. \& Karki B., 2015. Weather forecasting: traditional knowledge of the people of Uttarakhand Himalaya. J. Geogr. Environ. Earth Sci. Int., 3(3), 1-14, doi. org/10.9734/jgeesi/2015/19016

Retnowati A., Anantasari E., Marfai M.A. \& Dittmann A., 2014. Environmental ethics in local knowledge responding to climate change: an understanding of seasonal traditional calendar PranotoMongso and its phenology in karst area of gunung Kidul, Yogyakarta, Indonesia. Procedia Environ. Sci., 20, 785-794, doi. org/10.1016/j.proenv.2014.03.095

Reyes-García V. et al., 2019. A collaborative approach to bring insights from local observations of climate change impacts into global climate change research. Curr. Opin. Environ. Sustainability, 39, 1-8, doi.org/10.1016/j. cosust.2019.04.007

Rosenzweig C. et al., 2008. Attributing physical and biological impacts to anthropogenic climate change. Nature, 453, 353-357, doi.org/10.1038/nature06937

Sánchez-Cortés M.S. \& Chavero E.L., 2011. Indigenous perception of changes in climate variability and its relationship with agriculture in a Zoque community of Chiapas, Mexico. Clim. Change, 107, 363-389, doi. org/10.1007/s10584-010-9972-9

Son H.N., Chi D.T.L. \& Kingsbury A., 2019. Indigenous knowledge and climate change adaptation of ethnic minorities in the mountainous regions of Vietnam: a case study of the Yao people in Bac Kan Province. Agric. Syst., 176, 102683, doi.org/10.1016/j.agsy.2019.102683

Trochain J.-L., 1980. Écologie végétale de la zone intertropicale non désertique. Toulouse, France: Université Paul Sabatier.

Twumasi P.A., 2001. Social research in rural communities. Accra: Ghana Universities Press.

Udmale P. et al., 2014. Farmer's perception of drought impacts, local adaptation and administrative mitigation measures in Maharashtra State, India. Int. J. Disaster Risk Reduct., 10, 250-269, doi.org/10.1016/j. ijdrr.2014.09.011

White F., 1983. La végétation de l'Afrique. Recherches sur les ressources naturelles. Paris : ORSTOM-UNESCO, 384.

(54 réf.) 
Annexe. Liste des espèces utilisées comme marqueurs temporels dans la zone guinéenne du Togo - List of species used as temporal markers in the Guinean zone of Togo.

\begin{tabular}{|c|c|c|}
\hline $\mathbf{N}^{\circ}$ & Espèce végétale & Famille \\
\hline 1 & Abelmoschus esculentus (L.) Moench & Malvaceae \\
\hline 2 & Acacia auriculiformis Benth. & Fabaceae \\
\hline 3 & Adansonia digitata $\mathrm{L}$. & Malvaceae \\
\hline 4 & Albizia zygia (DC.) J.F.Macbr. & Fabaceae \\
\hline 5 & Alstonia boonei De Wild. & Apocynaceae \\
\hline 6 & Anacardium occidentale $\mathrm{L}$. & Anacardiaceae \\
\hline 7 & Andropogon gayanus Kunth & Poaceae \\
\hline 8 & Antiaris toxicaria Lesch. & Moraceae \\
\hline 9 & Aspilia rudis Oliv. \& Diern ssp. & Asteraceae \\
\hline 10 & Azadirachta indica A.Juss & Meliaceae \\
\hline 11 & Blighia sapida K.D.König & Sapindaceae \\
\hline 12 & Bridelia ferruginea Benth. & Euphorbiaceae \\
\hline 13 & Caesalpinia pulcherrima (L.) Sw. & Fabaceae \\
\hline 14 & Cajanus cajan (L.) Millsp. & Fabaceae \\
\hline 15 & Carica papaya $\mathrm{L}$. & Caryophyllaceae \\
\hline 16 & Ceiba pentandra (L.) Gaertn. & Bombacaceae \\
\hline 17 & Chamaecrista mimosoides (L.) Greene & Fabaceae \\
\hline 18 & Chromolaena odorata (L.) R.M.King \& H.Rob. & Asteraceae \\
\hline 19 & Citrus sinensis (L.) Osbeck & Rutaceae \\
\hline 20 & Clitoria falcata Lam. & Fabaceae \\
\hline 21 & Coffea arabica $\mathrm{L}$. & Rubiaceae \\
\hline 22 & Coffea canephora Pierre ex A.Froehner & Rubiaceae \\
\hline 23 & Cola gigantea A.Chev. & Sterculiaceae \\
\hline 24 & Combretum molle R.Br. ex G.Don & Combretaceae \\
\hline 25 & Cyperus squarrosus L. & Cyperaceae \\
\hline 26 & Daniellia oliveri (Rolfe) Hutch.\& Dalziel & Fabaceae \\
\hline 27 & Delonix regia (Hook.) Raf. & Fabaceae \\
\hline 28 & Dialium guineense Willd. & Caesalpiniaceae \\
\hline 29 & Dioscorea alata $\mathrm{L}$. & Dioscoreaceae \\
\hline 30 & Dioscorea cayennensis Lam. & Dioscoreaceae \\
\hline 31 & Elaeis guineensis Jacq. & Arecaceae \\
\hline 32 & Ficus exasperata Vahl & Moraceae \\
\hline 33 & Ficus glumosa DeliIe & Moraceae \\
\hline 34 & Garcinia kola Heckel & Clusiaceae \\
\hline 35 & Gardenia ternifolia Schumach. \& Thonn. & Rubiaceae \\
\hline 36 & Gliricidia sepium (Jacq.) Walp. & Fabaceae \\
\hline 37 & Gmelina arborea Roxb. & Verbenaceae \\
\hline 38 & Hyptis suaveolens (L.) Poit. & Lamiaceae \\
\hline 39 & Indigofera hirsuta $\mathrm{L}$. & Fabaceae \\
\hline 40 & Irvingia gabonensis (Aubry-Lecomte ex O'Rorke) Baill. & Inrvingiaceae \\
\hline
\end{tabular}


Annexe (suite). Liste des espèces utilisées comme marqueurs temporels dans la zone guinéenne du Togo - List of species used as temporal markers in the Guinean zone of Togo.

\begin{tabular}{|c|c|c|}
\hline $\mathbf{N}^{\circ}$ & Espèce végétale & Famille \\
\hline 41 & Jatropha curcas L. & Euphorbiaceae \\
\hline 42 & Khaya senegalensis (Desv.) A.Juss. & Meliaceae \\
\hline 43 & Launaea taraxacifolia (Willd.) Amin ex C.Jeffrey & Asteraceae \\
\hline 44 & Lonchocarpus sericeus (Poir.) DC. & Fabaceae \\
\hline 45 & Mangifera indica $\mathrm{L}$. & Anacardiaceae \\
\hline 46 & Milicia excelsa (Welw.) C.C.Berg & Moraceae \\
\hline 47 & Millettia thonningii (Schum. \& Thonn.) Baker & Fabaceae \\
\hline 48 & Morinda lucida Benth. & Rubiaceae \\
\hline 49 & Moringa oleifera Lam. & Moringaceae \\
\hline 50 & Mucuna poggei Taub. & Fabaceae \\
\hline 51 & Newbouldia laevis (P.Beauv.) Seem. & Bignoniaceae \\
\hline 52 & Parkia biglobosa (Jacq.) G.Don & Fabaceae \\
\hline 53 & Persea americana Mill. & Lauraceae \\
\hline 54 & Pupalia lappacea (L.) Juss. & Amaranthaceae \\
\hline 55 & Senna occidentalis (L.) Link & Fabaceae \\
\hline 56 & Senna siamea (Lam.) H.S.Irwin \& Barneby & Fabaceae \\
\hline 57 & Spathodea campanulata P.Beauv. & Bignoniaceae \\
\hline 58 & Spondias mombin L. & Anacardiaceae \\
\hline 59 & Tectona grandis L.f. & Verbenaceae \\
\hline 60 & Tephrosia elegans Schum. & Fabaceae \\
\hline 61 & Terminalia mollis M.A.Lawson & Combretaceae \\
\hline 62 & Theobroma cacao L. & Sterculiaceae \\
\hline 63 & Tridax procumbens (L.) L. & Asteraceae \\
\hline 64 & Triumfetta rhomboidea Jacq. & Tiliaceae \\
\hline 65 & Vernonia amygdalina Del. & Asteraceae \\
\hline 66 & Vitellaria paradoxa C.F.Gaertn. & Sapotaceae \\
\hline 67 & Vitex doniana Sweet & Verbenaceae \\
\hline
\end{tabular}

